\title{
INCIDENCE RINGS WITH SELF-DUALITY
}

\author{
JOEL K. HAACK
}

\begin{abstract}
ABSTRACr. An artinian ring $R$ is said to have self-duality if there is a Morita duality between the categories of left and right finitely generated $R$-modules. Here it is shown that the incidence ring of a finite preordered set over a division ring has self-duality. This is accomplished in part by calculating their injective modules.
\end{abstract}

Theorems of Azumaya [3], Morita [11], and Tachikawa [13] in the late 1950's give necessary and sufficient conditions to insure that the category of finitely generated left modules over a (necessarily left artinian) ring $R$ is dual to the category of finitely generated right modules over a ring $S$. An open question in the subject of Morita duality for artinian rings is that of characterizing those artinian rings, other than artin algebras and QF rings, that have self-duality, that is, those for which there exists a duality between their categories of left and right finitely generated modules. Recently, artinian rings with self-duality have been shown to include certain factors of skew polynomial rings [12], hereditary artinian tensor rings satisfying the Dlab-Ringel duality conditions [2], rings with quivers that are trees [8] and many serial rings [9]. Here we prove that the incidence ring of a finite preordered set over a division ring (hereafter called a finite incidence ring) has self-duality. This class of rings properly contains the class of hereditary serial rings and, indeed, all artinian rings with quivers that are trees [8]. The self-duality constructed is weakly symmetric, so we may apply $[9,(4.1)]$ to show that any factor ring of a finite incidence ring has a (weakly symmetric) self-duality.

A finite incidence ring over a division ring $D$ can be characterized as a (unital) subring of the $(n \times n)$-matrix ring over $D$ satisfying $R=\Sigma\left\{D I_{k l} \mid I_{k k} R I_{l l} \neq 0\right\}$, where $I_{k l}$ is the matrix unit with 1 in the $(k, l)$-position and 0 elsewhere. (Thus, finite incidence rings coincide with Mitchell's tic tac toe rings over division rings [10].) To prove that an artinian ring $R$ has self-duality, it is sufficient to show that the basic ring $e R e$ of $R$ is isomorphic to the endomorphism ring of the minimal left injective cogenerator over $e \operatorname{Re}$ [3], [11]. It is not hard to show that the basic ring of a finite incidence ring is a finite incidence ring over the same division ring. Henceforth, we let $R$ be a basic indecomposable $(n \times n)$-finite incidence ring over the division ring $D$. We shall consider $D$ as the subring of constant diagonal matrices in $R$. Let $e_{k}=I_{k k}$ be the matrix unit with 1 in the $(k, k)$-position and 0 elsewhere. Then $e_{k} \in R$. If $I_{k l} \in R$, let $e_{k l}=I_{k l}$; if not, let $e_{k l}=0$. The radical of $R$ is

$$
J=J(R)=\sum\left\{D e_{i j} \mid i \neq j\right\} .
$$

Received by the editors March 13, 1979.

AMS (MOS) subject classifications (1970). Primary 16A46, 16A49; Secondary 16A42, 16A52.

Key words and phrases. Morita duality, incidence rings, injective modules. 
We denote the composition length of a module $N$ by $c(N)$ and its injective envelope by $E(N)$. Let $M$ be the set of $(n \times 1)$-column vectors over $D$, with a left $R$-structure given by considering $R$ as a subring of the $(n \times n)$-matrix ring over $D$. Let $m_{k}=\left(\delta_{k, i}\right)_{i} \in M$. Define a monomorphism $\iota_{k}: R e_{k} \rightarrow M$ by $\iota_{k}: r e_{k} \mapsto r e_{k} m_{k}=$ $r m_{k}$. Since $c\left({ }_{e_{k} R e_{k}} e_{k} M\right)=1$, we conclude from [4] that $M$ and hence also $\operatorname{Re}_{k}$ are distributive (have a distributive lattice of submodules) for all $k$. Similarly, $e_{k} R$ is distributive for each $k$.

Now consider any submodule $N$ of $M$, and let $x \in N$ with $x=\sum_{k=1}^{n} d_{k} m_{k}$. If $d_{k} \neq 0$, then $e_{k} d_{k}^{-1} x=e_{k} d_{k}^{-1} d_{k} m_{k}=m_{k}$, so $m_{k} \in N$. Thus $x=\sum_{k=1}^{n} d_{k} m_{k}=$ $\sum_{m_{k} \in N} d_{k} m_{k}$, so

$$
N=\sum\left\{D m_{k} \mid m_{k} \in N\right\}
$$

Define submodules $L_{k} \subset M$ by $L_{k}=\sum\left\{R m_{j} \mid e_{k} R e_{j}=0\right\}$. If $e_{k} R e_{j} \neq 0$, then $e_{k j} \neq 0$; thus $e_{k j}=0$ implies $m_{j} \in L_{k}$. Conversely, suppose that $m_{j} \in L_{k}$ and write $m_{j}=\sum r_{i} m_{i}$ with $e_{k i}=0$ and $r_{i} \in R$. Then $e_{j} m_{j}=\sum e_{j} r_{i} m_{i}$, so there exists $i$ with $e_{j} r_{i} e_{i} m_{i} \neq 0$ and $e_{k i}=0$. Thus $e_{j} r_{i} e_{i} \neq 0$, so $e_{j i} \neq 0$. But now if $e_{k j} \neq 0$, then $0 \neq e_{k j} e_{j i}$ and $e_{k i} \neq 0$, a contradiction. Hence $m_{j} \in L_{k}$ implies $e_{k j}=0$, and

$$
L_{k}=\sum\left\{D m_{j} \mid e_{k j}=0\right\}
$$

Let $E_{k}=M / L_{k}$ and let $\eta_{k}$ be the natural epimorphism.

1. Proposition. The module $E_{k}=M / L_{k}$ is the injective envelope of $R e_{k} / J e_{k}$.

Proof. We first show that $\operatorname{Soc}\left(E_{k}\right) \cong R e_{k} / J e_{k}$. Let $x=\Sigma d_{j} m_{j}+L_{k}$ be any nonzero element of $E_{k}$ and suppose that $d_{i} \neq 0$ with $i \neq k$ and $e_{k i} \neq 0$. Then $0 \neq d_{i} m_{k}+L_{k}=e_{k i} \sum d_{j} m_{j}+L_{k} \in J x$. Therefore $x \notin \operatorname{Soc}\left(E_{k}\right)$. So $e_{i} \operatorname{Soc}\left(E_{k}\right)=0$ if $e_{k i} \neq 0$ and $k \neq i$, and of course $e_{i} \operatorname{Soc}\left(E_{k}\right)=0$ if $e_{k i}=0$ (so that $m_{i} \in L_{k}$ ). Since $\operatorname{Soc}\left(E_{k}\right) \neq 0$, we must have $e_{k} \operatorname{Soc}\left(E_{k}\right) \neq 0$. Since $M$ is distributive, so also is $E_{k}$; thus $\operatorname{Soc}\left(E_{k}\right) \cong R e_{k} / J e_{k}$. To conclude we may apply [7, Lemma 5] and [6, Lemma 2.3] to see that the lattice of submodules of $E\left(R e_{k} / J e_{k}\right)$ is isomorphic to that of $e_{k} R_{R}$. Hence

$$
\begin{aligned}
c\left(E\left(R e_{k} / J e_{k}\right)\right) & =c\left(e_{k} R_{R}\right)=\sum_{j=1}^{n} c\left(e_{k} R e_{j e_{j} R e_{j}}\right) \\
& =\#\left\{j \mid e_{k} R e_{j} \neq 0\right\}=n-\#\left\{j \mid e_{k} R e_{j}=0\right\}=c\left(E_{k}\right) .
\end{aligned}
$$

Because $\operatorname{Soc}\left(E_{k}\right) \cong R e_{k} / J e_{k}, E_{k}$ is a submodule of $E\left(R e_{k} / J e_{k}\right)$ of the same length as $E\left(R e_{k} / J e_{k}\right)$, so $E_{k}=E\left(R e_{k} / J e_{k}\right)$.

Next we show that $\operatorname{End}(M)$ is a division ring.

2. Proposition. Let $g: M \rightarrow M$.

(1) If ker $g \neq 0$ then $g=0$.

(2) If im $g \neq M$ then $g=0$.

Thus $\operatorname{End}(M)$ is a division ring. Moreover, $\operatorname{End}(M) \cong D$. 
Proof. If ker $g \neq 0$, choose $e_{j_{0}}$ so that $e_{j_{0}} \operatorname{ker} g \neq 0$, i.e., so that $m_{j_{0}} \in$ ker $g$. Let $m_{h}$ be given. We will show that $m_{h} g=0$. Since $R$ is indecomposable, there exist $e_{i}, \ldots, e_{i_{1}}$ and $e_{j_{1}}, \ldots, e_{j_{l}}=e_{h}$ such that $e_{j_{k-1}} R e_{i_{k}} \neq 0 \neq e_{j_{k}} R_{i_{i_{k}}}$ for $k=1, \ldots, l$. (See $[1, \S 7]$.) Suppose that $m_{j_{k-1}} g=0$. Then also $m_{i_{k}} g=0$, for if $m_{i_{k}} g \neq 0$, then $0 \neq$ $e_{j_{k-1} i_{k}} e_{i_{k}}\left(m_{i_{k}} g\right)=m_{j_{k-1}} g$. And now $m_{j_{k}} g=\left(e_{j_{k} i_{k}} m_{i_{k}}\right) g=e_{j_{k} i_{k}}\left(m_{i_{k}} g\right)=0$. Thus by induction $m_{h} g=0$ for all $h$ and $M g=0$.

For (2), note that if im $g \neq M$, then $\operatorname{ker} g \neq 0$, so by (1), $g=0$.

For the moreover part, let $f: M \rightarrow M$ and let $S=R m_{k}$ be a simple submodule of $M$. Since $M$ is distributive, $\operatorname{Soc}(M)$ is square-free so $S f \subset S$. Thus we may regard $\left.f\right|_{S}: S \rightarrow S$ as an $e_{k} R e_{k}$-map. But then for some $d \in D,\left.d_{k} m_{k} f\right|_{S}=d_{k} d m_{k}$ for all $d_{k} m_{k} \in S$. Let $f^{\prime}: M \rightarrow M$ via $f^{\prime}: m \mapsto m d$. Then $0 \neq S \subset \operatorname{ker}\left(f^{\prime}-f\right)$, so by (1), $f^{\prime}=f$ and the ring monomorphism $\Phi: D \rightarrow \operatorname{End}(M)$ via $\Phi(d): m \mapsto m d$ is onto. Hence $D \cong \operatorname{End}(M)$.

In our proof that finite incidence rings have self-duality, the main technique is that of changing the range or domain of a function. For example, if $N$ is a distributive artinian module, $L$ is a submodule of $N$ and $f$ is a map $f: L \rightarrow N$, then im $f \subset L$, so we may regard $f$ as a map from $L$ to $L$. Dually, if $N$ is a distributive noetherian module, $L$ is a submodule of $N$ and $f$ is any map $f: N \rightarrow N / L$, then ker $f \supset L$, so we may regard $f$ as a map from $N / L$ to $N / L$ by the factor theorem. (See $[5, \S 4.1]$.) These results allow us to develop the principal tool used in the proof of Theorem 4.

3. Lemma. (1) Let $L$ be a nonzero indecomposable submodule of $M$ and let $f$ : $L \rightarrow M$. Then there exists a unique map $f^{\prime}: M \rightarrow M$ such that $l^{\prime}=$ lf for all $l \in L$.

(2) Let $L=M / K$ be a nonzero indecomposable factor of $M$ and let $f: M \rightarrow L$. Then there exists a unique map $f^{\prime}: M \rightarrow M$ such that $m f^{\prime}+K=m f$ for all $m \in M$.

Proof. (1) Let $L$ be a nonzero indecomposable submodule of $M$ and let $e=\Sigma\left\{e_{j} \mid e_{j} L \neq 0\right\}$. Since $c\left(e_{j} R e_{j} e_{j} M\right)=1$ for all $j$, either $e_{j} L=0$ or $e_{j} L=e_{j} M$. Thus $L=e M$. Let $f: L \rightarrow M$. By the remarks preceding the lemma, $L f \subset L$. Let $f^{*}$ denote $f$ with range restricted to $L$. Now $e R e$ is a finite incidence ring over $D$, $L=e M$ plays the role of $M$ for $e R e$ and $f^{*} \in \operatorname{End}(e R e M)$. Since $e M$ is indecomposable over $R$, it is an indecomposable module over $e R e$. Since $e M$ is also faithful over $e R e, e R e$ is an indecomposable ring and we may apply Proposition 2 to see that $f^{*}$ is right multiplication by some $d \in D$. Define $f^{\prime}: M \rightarrow M$ via $f^{\prime}: m \mapsto m d$. Then $f^{\prime}$ extends $f$. If $g: M \rightarrow M$ also extends $f$, then $0 \neq L \subset \operatorname{Ker}\left(g-f^{\prime}\right)$, so by Proposition 2, $g=f^{\prime}$ and $f^{\prime}$ is unique.

(2) Let $L=M / K$ be a nonzero indecomposable submodule of $M$, let $e=$ $\Sigma\left\{e_{j} \mid e_{j} L \neq 0\right\}$ and let $f: M \rightarrow L$. By the remarks preceding the lemma, $K \subset \operatorname{ker} f$, so we may define a map $f^{*}: L \rightarrow L$ via $f^{*}: m+K \mapsto m f$. Now $e R e$ is a finite incidence ring over $D, L=e(M / K)$ plays the role of $M$ for $e R e$ and $f^{*} \in$ End $\left({ }_{e R e} L\right)$. Since $L$ is indecomposable over $R$, it is an indecomposable module over $e R e$. Since $L$ is also faithful over $e R e, e R e$ is indecomposable and we may apply Proposition 2 to see that for some $d \in D,(m+K) f^{*}=m d+K$ for all $m \in M$. Define $f^{\prime}: M \rightarrow M$ via $f^{\prime}: m \mapsto m d$. Then $m f^{\prime}+K=m d+K=(m+K) f^{*}=m f$ 
for all $m \in M$. If $g: M \rightarrow M$ also satisfies $m g+K=m f$ for all $m \in M$, then $m\left(g-f^{\prime}\right) \in K$ for all $m$, so $\operatorname{im}\left(g-f^{\prime}\right) \neq M$. Thus by Proposition $2, g=f^{\prime}$.

A duality $D^{\prime}$ between the categories of left and right finitely-generated $R$-modules is said to be weakly symmetric if for $J=\operatorname{rad}(R)$ and for $e$ any primitive idempotent of $R, D^{\prime}(R e / J e) \cong e R / e J$. It is not hard to see that a ring isomorphism $\Phi: R \rightarrow \operatorname{End}\left({ }_{R} E\right)$ such that $E \Phi(e)=E(R e / J e)$, for each primitive idempotent $e \in R$, induces such a weakly symmetric duality if $R$ is artinian and ${ }_{R} E$ is an injective cogenerator [9, (3.1)].

4. THEOREM. Let $R$ be a finite incidence ring over a division ring. Then $R$ has $a$ weakly symmetric duality.

Proof. We may assume that $R$ is basic and indecomposable. Let the $R$-module $M$ be defined as it has been throughout. Let $\iota_{i}: R e_{i} \rightarrow M$ be the natural monomorphism and $\eta_{i}: M \rightarrow M / L_{i}=E_{i}$ be the natural epimorphism as before. Let $E=$ $\bigoplus_{i=1}^{n} E_{i}$ be the minimal injective cogenerator of $R$-mod and let $S=\operatorname{End}\left({ }_{R} E\right)$ with $f_{i} \in S$ the natural projection onto $E_{i}$. Define $\theta: S \rightarrow R$ via $\theta: \sum f_{i} s f_{j} \mapsto \sum e_{i} r e_{j}$, where $e_{i} r e_{j}$ is defined below.

Consider the following commutative diagram:

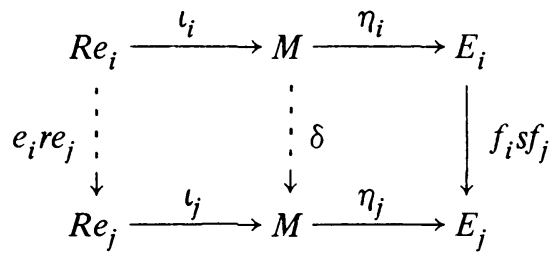

By Lemma 3 there exists a unique $\delta: M \rightarrow M$ such that $n_{i} f_{i} s f_{j}=\delta \eta_{j}$. If $f_{i} s f_{j}=0$, then $\delta=0$ and $e_{i} r e_{j}=0$ is the only choice for $e_{i} r e_{j}$ to make the diagram commute. If $f_{i} s f_{j} \neq 0$, then $e_{j} E_{i} \neq 0$, so also $0 \neq e_{i} R e_{j}=\operatorname{Hom}\left(R e_{i}, R e_{j}\right)$ [6, Theorem 2.4]. Thus $e_{i j} \neq 0$, so $m_{i}=e_{i j} m_{j}$ and im $\iota_{i} \delta \subset R m_{i} \subset R m_{j}$. Therefore the map $e_{i} r e_{j}$ exists uniquely with $e_{i} r e_{j} \iota_{j}=\iota_{i} \delta$. Thus, $\theta$ is a well-defined function. To see that $\theta$ is bijective, let $e_{i} r e_{j} \neq 0$ be given. By Lemma 3 there exists a unique $\delta: M \rightarrow M$ such that $e_{i} r e_{j} \iota_{j}=\iota_{i} \delta$. Now $e_{i} r e_{j} \neq 0$ implies that $e_{i j} \neq 0$, so that if $e_{j k} \neq 0$ then also $e_{i k}=e_{i j} e_{j k} \neq 0$. Thus $e_{i k}=0$ implies $e_{j k}=0$, so $L_{i} \subset L_{j}$. Since $M$ is distributive, $L_{i} \delta \subset L_{i} \subset L_{j} \subset \operatorname{Ker} \delta \eta_{j}$. Hence $\delta \eta_{j}$ factors uniquely through $\eta_{i}$. Let $\eta_{i} f_{i} s i_{j}=\delta \eta_{j}$. Thus $\theta$ is bijective.

A simple argument shows that $\delta+\delta^{\prime}$ and $e_{i} r e_{j}+e_{i} r^{\prime} e_{j}$ are the maps associated with $f_{i} s f_{j}+f_{i} s^{\prime} f_{j}$, and it follows that $\theta$ is additive. Since for each $i, j, k \in$ $\{1, \cdots, n\}, f_{i} s f_{j} s^{\prime} f_{k}$ corresponds to $\delta \delta^{\prime}$ and then to $e_{i} r e_{j} r^{\prime} e_{k}, \theta$ is multiplicative. Thus $\theta$ is a ring isomorphism. Also, $f_{i}$ corresponds to $1_{M}$ corresponds to $e_{i}$, so $\theta\left(f_{i}\right)=e_{i}$, and $R$ has a weakly symmetric duality.

If $R$ is an artinian ring with a weakly symmetric duality and the primitive right (or left) ideals of $R$ are distributive, then any factor ring of $R$ has a weakly symmetric duality $[9,(4.1)]$. Since the primitive right (and left) ideals of a finite incidence ring are distributive, we may apply Theorem 4 to conclude that factor rings of finite incidence rings also have self-duality. 
5. Corollary. Any factor ring of a finite incidence ring over a division ring has a weakly symmetric duality.

We note here that a finite incidence ring over a division ring is a tensor ring iff it is hereditary, and an artinian tensor ring is a finite incidence ring iff every principal right and left ideal generated by a primitive idempotent is distributive. Nonhereditary finite incidence rings are nontrivial factors of tensor rings with the same quivers, but since such tensor rings do not satisfy the hypotheses of [9, (4.1)], one cannot apply a result of Auslander, Platzeck, and Reiten [2], namely, that hereditary artinian tensor rings satisfying the Dlab-Ringel duality conditions have selfduality, to show directly that finite incidence rings have self-duality.

Acknowledgement. This paper constitutes a portion of a Ph.D. thesis written under the supervision of Professor K. R. Fuller and submitted to the graduate faculty of the University of Iowa. The author wishes to express his gratitude to Professor Fuller for his encouragement and helpful suggestions.

\section{REFERENCES}

1. F. W. Anderson and K. R. Fuller, Rings and categories of modules, Graduate Texts in Math., Vol. 13, Springer-Verlag, Berlin and New York, 1974.

2. M. Auslander, M. I. Platzeck and I. Reiten, Coxeter functors without diagrams, Trans. Amer. Math. Soc. 250 (1979), 1-46.

3. G. Azumaya, A duality theory for injective modules, Amer. J. Math. 81 (1959), 249-278.

4. V. P. Camillo, Distributive modules, J. Algebra 36 (1975), 16-25.

5. P. M. Cohn, Free rings and their relations, Academic Press, London and New York, 1971.

6. K. R. Fuller, On indecomposable injectives over Artinian rings, Pacific J. Math. 29 (1969), 115-135.

7. __ Rings of left invariant module type, Comm. Algebra 6 (1978), 153-167.

8. K. R. Fuller and J. Haack, Rings with quivers that are trees, Pacific J. Math. 76 (1978), 371-379.

9. J. K. Haack, Self-duality and serial rings, J. Algebra 59 (1979), 345-363.

10. B. Mitchell, Theory of categories, Academic Press, New York and London, 1965.

11. K. Morita, Duality of modules and its applications to the theory of rings with minimum condition, Sci. Rep. Tokyo Kyoiku Daigaku 6 (1958), 85-142.

12. B. Roux, Modules injectifs indécomposables sur les anneaux artiniens et dualité de Morita, Sém. P. Dubreil (26e année 1972/73), Algèbre, Exp. No. 10, Secrétariat Mathématique, Paris, 1973, 19 pp.

13. H. Tachikawa, Duality theorem of character modules for rings with minimum condition, Math. Z. 68 (1958), 479-487.

Department of Mathematics, The University of Iowa, Iowa City, Iowa 52242

Current address: Department of Mathematics, Oklahoma State University, Stillwater, Oklahoma 74074 